\title{
PROJETO DE INSTALAÇÃO DE PRODUÇÃO INDUSTRIAL DE CARBONATO DE GLICERINA
}

\author{
B. P. CELIBERTO ${ }^{1}$, C. C. FICO ${ }^{1}$, G. RUSSO ${ }^{1}$, L. L. NAME ${ }^{1}$, V. M. B. BARROS ${ }^{1}$, \\ N. L. FERREIRA, ${ }^{1, *}$ \\ ${ }^{1}$ Centro Universitário da FEI, Departamento de Engenharia Química \\ *E-mail: nlibanio@fei.edu.br
}

\begin{abstract}
RESUMO: Indústrias de biodiesel geram grandes quantidades de glicerina, o que torna necessária a proposição de uma destinação viável e lucrativa para esse subproduto. Para dar destino à abundância da glicerina produzida, muitos pesquisadores e indústrias vêm purificando-a ou convertendo-a em produtos químicos valiosos, tais como carbonato de glicerina. $O$ carbonato de glicerina é uma substância de ampla aplicação industrial, na indústria cosmética e de fármacos, na produção de polímeros, na utilização como solvente, entre outros. Este trabalho visa o estudo da produção de carbonato de glicerina capaz de atingir altas conversões, próximas a 100\%, sendo avaliada a viabilidade de implementação de reciclo e tanque flash. Para a realização do mesmo foi utilizado o software Aspen Plus ${ }^{\circledR}$, com o intuito da aplicação em escala industrial da transesterificação catalítica da glicerina, sendo feita também a análise de custos de reagentes e insumos, como água de resfriamento e vapor. A partir da obtenção de uma via simples de produzir carbonato de glicerina, a modelagem proposta neste trabalho foi baseada no modelo termodinâmico NRTL e em uma cinética de reação que pode atingir $98 \%$ de pureza, praticamente isento dos possiveis reagentes tóxicos que participam da reação.
\end{abstract}

PALAVRAS-CHAVE: Glicerina; Transesterificação; Catálise; Carbonato de glicerina.

\section{INTRODUÇÃO}

Com o crescente desenvolvimento de métodos de produção de biodiesel também aumenta o estudo de métodos de aproveitamento do seu subproduto, a glicerina. Apesar da grande quantidade de usos para a glicerina, como por exemplo, aplicações farmacêuticas, uso em cosméticos, uso alimentício, síntese de resinas, entre outros, existe um excedente de matéria-prima, e que cresce cada vez mais sua produção com o biodiesel (BIODIESELBR, 2006).

O carbonato de glicerina é um precursor de polímeros, emulsificantes alimentares, tintas, detergentes, perfumes e cosméticos, entre outros (OCHOA-GÓMEZ et al. 2012). Pode ser sintetizado pelo uso de glicerol como matéria-prima. Comprovou-se que existem altas taxas de conversões utilizando dimetilcarbonato (DMC), e carbonato de potássio como 
catalisador (ESTEBAN et al. 2015), além de estipulação da cinética da reação. Outros métodos foram estudados, como o uso de micro-ondas e glicerina bruta, para análise de rendimento às diversas temperaturas, concentração de catalisador e efeitos das impurezas na reação (TENG et al., 2016).

Esteban et al. (2015) derivou a cinética da reação, o que possibilita um estudo em reatores para controle de temperatura e tempo de residência para a reação proposta. Pode-se ainda analisar o fato de que componentes vistos como impurezas na glicerina bruta têm, em sua maioria, uma nova interpretação como solventes ou catalisadores do processo. As características do reagente, portanto, podem garantir redução de custos na produção de carbonato de glicerina (GC) devido à falta de necessidade de purificação de um dos reagentes.

Este trabalho tem como objetivo a proposição de um processo produtivo de GC e a análise econômica preliminar de um caso de produção industrial, utilizando glicerina como matéria-prima. É necessário o estudo da reação envolvida e estipulação de reator e operações unitárias envolvidas. Serão feitas simulações no programa Aspen Plus ${ }^{\circledR}$ para análise do processo que envolverá comportamento dos compostos nas operações de separação e complementares e a cinética de reação.

\section{MATERIAIS E MÉTODOS}

Neste trabalho foi estudada a produção de carbonato de glicerina em escala industrial a partir da transesterificação do dimetilcarbonato com glicerina, com um catalisador de natureza básica, segundo o procedimento de Esteban et al. (2015), bem como o impacto produtivo e/ou econômico que certas mudanças podem exercer sobre o sistema, como, por exemplo, a utilização de glicerina pura ou bruta, a relevância de criação de reciclos e as possíveis retiradas de produtos secundários para venda.

Foi utilizado um fluxograma baseado no processo descrito no artigo de Ochoa-gómez et al. (2012), com mudanças devido às diferenças de projeto, tais como reagentes, catalisadores, e outras que foram julgadas necessárias.

Utilizando o software Aspen Plus®, foram feitas variações e testes do fluxograma de processo para que ficasse de acordo com os nossos objetivos, além de verificar a viabilidade em utilizar a glicerina bruta ao invés da glicerina pura. $\mathrm{O}$ modelo termodinâmico adotado na simulação é o NRTL, uma vez que este método considera que o sistema é extremamente não ideal, porém ele não possui todos os parâmetros que podem ser necessários para realizar as simulações, sendo então necessária a complementação pelo método termodinâmico UNIFAQ. O modelo NRTL é mais rigoroso no cálculo das propriedades termodinâmicas necessárias para o estudo, por isso foi a escolha principal para o cálculo das mesmas. Para a convergência do modelo com reciclo usou-se o método de Broyden, disponível no software.

Para a modelagem do reator foi utilizada uma das cinéticas presentes em Esteban et al. (2015), que são: primeira ordem, segunda ordem e uma cinética que combina duas equações que descrevem um comportamento da mistura em fase heterogênea e homogênea, sendo estas separadas por uma conversão crítica igual a 0,3 (conversão esta que gera metanol o suficiente para atuar como solvente para a reação estudada). Foi escolhido o terceiro modelo 
matemático, descartando a primeira lei cinética que compõe o conjunto, uma vez que o sistema já inicia a reação com solvente o suficiente para que haja homogeneidade do meio. A cinética segue na Equação (1) abaixo:

$$
r=k_{0} \exp \left(-\frac{E_{a}}{R T}\right) C_{c a t} C_{G L Y} C_{D M C}
$$

Onde: $\mathrm{k}_{0}=1,183 \times 10^{24}\left(\mathrm{~L}^{2} \cdot \mathrm{mol}^{-2} \cdot \mathrm{s}^{-1}\right), \mathrm{E}_{\mathrm{a}}=179,233\left(\mathrm{~kJ} \cdot \mathrm{mol}^{-1}\right), \mathrm{C}_{\text {cat }}=$ concentração de catalisador $\left(\mathrm{mol} \cdot \mathrm{L}^{-1}\right), \mathrm{C}_{\mathrm{GLY}}=$ concentração de glicerina $\left(\mathrm{mol} \cdot \mathrm{L}^{-1}\right)$ e $\mathrm{C}_{\mathrm{DMC}}=$ concentração de DMC.

Ao longo do trabalho foi necessária a troca do método de tratamento das substâncias, que não poderia ser simplesmente o convencional, devido a inconsistências observadas nas colunas de destilação (apresentadas nos itens seguintes).

A mudança foi, basicamente, a troca da classe das correntes nos ajustes de simulação. O simulador trata em geral as suas correntes com o modelo "convencional" que considera as substâncias químicas em estado líquido geralmente e apesar de permitir o tratamento de sólidos, apresenta irregularidades em cálculos de determinadas operações unitárias. A nova classe MIXCISLD traz uma nova aproximação, onde as substâncias apresentadas na aba "componentes" também podem se comportar como sólidos que não participarão diretamente de certas operações. Esta mudança permite por exemplo que o simulador desconsidere a necessidade de um sólido ser "evaporado" em um tanque flash, por exemplo.

A análise da viabilidade econômica será feita a partir da comparação de índices de matéria prima, vapor para aquecimento e água de resfriamento utilizado na produção, e do custo de massa de produto de cada um dos três processos analisados, que são o processo sem reciclo, com reciclo e com reciclo e tanque flash. $O$ índice da matéria prima $\left(\mathrm{I}_{\mathrm{MP}}\right)$ foi calculado a partir da Equação (2):

$$
I_{M P}=\frac{\sum C R \cdot m_{R}}{m_{P F}}
$$

Onde: $\mathrm{CR}=$ custo dos reagentes $\left(\mathrm{R} \$ \mathrm{~kg}^{-1}\right) ; \mathrm{m}_{\mathrm{R}}=$ massa de reagente $(\mathrm{kg})$ e $\mathrm{m}_{\mathrm{PF}}=$ massa de produto formado $(\mathrm{kg})$

Para a determinação do índice relativo à água de resfriamento foi necessário o cálculo da massa usada para refrigerar todos os resfriadores existentes no processo. Para isso, foi utilizado a equação do calor sensível que aquecerá a água de refrigeração, e o calor Qs é um dado fornecido pela tabela de resultados do simulador.

$$
Q_{S}=m_{\text {aref }} c_{p} \Delta T
$$

Onde: $\mathrm{Q}_{\mathrm{S}}=$ calor sensível $\left(\mathrm{kJ} \cdot \mathrm{h}^{-1}\right), m_{\text {aref }}=$ vazão mássica de água de refrigeração $\left(\mathrm{kg} \cdot \mathrm{h}^{-1}\right), \mathrm{c}_{\mathrm{p}}$ = calor específico $\left(\mathrm{kJ} \cdot \mathrm{kg}^{-1} \cdot{ }^{\circ} \mathrm{C}^{-1}\right)$ e $\Delta \mathrm{T}=$ diferença de temperatura $\left({ }^{\circ} \mathrm{C}\right)$. 
O valor do calor específico $\left(\mathrm{c}_{\mathrm{p}}\right)$ utilizado foi $0,24\left(\mathrm{~kJ} \cdot \mathrm{kg}^{-1} \cdot{ }^{\circ} \mathrm{C}^{-1}\right)$, uma que vez que foi considerado que a variação do $c_{p}$ da água para as condições propostas é insignificante para a estimativa preliminar de gastos. A variação da temperatura utilizada foi de $15{ }^{\circ} \mathrm{C}$, sendo a temperatura de entrada da água no resfriador de $30^{\circ} \mathrm{C}$, que seria a máxima temperatura que a água poderia chegar no pior caso possível, e a temperatura que a água sai do resfriador foi considerada como $45^{\circ} \mathrm{C}$, que é a máxima temperatura que a água pode ser devolvida ao meio ambiente sem que exista danos a este. $\mathrm{O}$ índice de água de resfriamento $\left(\mathrm{I}_{\mathrm{AR}}\right)$ foi calculado a partir da seguinte relação (Equação 4):

$$
I_{A R}=\frac{\sum Q_{S}}{m_{P F} c_{p} \Delta T}
$$

Onde: $\mathrm{QS}_{\mathrm{S}}=$ calor sensível $\left(\mathrm{kJ} \cdot \mathrm{h}^{-1}\right), \mathrm{m}_{\mathrm{PF}}=$ vazão mássica de produto formado $\left(\mathrm{kg} \cdot \mathrm{h}^{-1}\right)$

A determinação do índice de vapor utilizado segue o mesmo princípio, com a diferença de que o calor trocado pelos aquecedores é o calor latente. A equação do calor latente utilizada para o cálculo da massa de vapor de aquecimento é realizado utilizando a Equação (5):

$$
Q_{L}=m_{v a p} h_{v a p}
$$

Onde: $\mathrm{Q}_{\mathrm{L}}=$ calor latente $\left(\mathrm{kJ} \cdot \mathrm{h}^{-1}\right), m_{\text {vap }}=$ vazão mássica de vapor $\left(\mathrm{kg} \cdot \mathrm{h}^{-1}\right)$ e $\mathrm{h}_{\mathrm{vap}}=$ entalpia de vaporização $\left(\mathrm{kJ} \cdot \mathrm{kg}^{-1}\right)$

Os calores sensíveis das transformações de vapor superaquecido para vapor saturado, ou de líquido saturado para líquido subresfriado são insignificantes quando comparados ao valor numérico do calor latente, e por isso optou-se em utilizar somente um vapor saturado com temperatura de aproximadamente $240^{\circ} \mathrm{C}$ e pressão de $34 \mathrm{kgf} / \mathrm{cm}^{2}$. A entalpia de vaporização para este vapor possui o valor de 1764,6 kJ/kg (SMITH et al. 2007). O índice de vapor de aquecimento (Iv) foi calculado a partir da Equação (6):

$$
I_{V}=\frac{\sum Q_{L}}{m_{P F} h_{v a p}}
$$

Onde: $\mathrm{Q}_{\mathrm{L}}=$ calor latente $\left(\mathrm{kJ} \cdot \mathrm{h}^{-1}\right) ; m_{\text {vap }}=$ vazão mássica de vapor $\left(\mathrm{kg} \cdot \mathrm{h}^{-1}\right)$ e $\mathrm{h}_{\text {vap }}=$ entalpia de vaporização $\left(\mathrm{kJ} \cdot \mathrm{kg}^{-1}\right)$.

\section{RESULTADOS E DISCUSSÃO}

\subsection{Uso do Metanol como Agente de Partida}

Para o processo de produção do carbonato de glicerina é necessário a adição de metanol para homogeneizar o meio reacional, porém o mesmo também é produto da reação em estudo, e, conforme a reação acontece, o metanol vai sendo formado, auxiliando na homogeneização da mistura. Para dar partida no reator e iniciar a reação seria necessária uma 
alimentação inicial de metanol por um curto período, até que a reação atinja sua conversão crítica (ESTEBAN et al. 2015), sendo posteriormente cortada essa adição. Contudo, o software Aspen Plus ${ }^{\circledR}$ calcula o final do processo, já em regime permanente, então, optou-se por não demostrar sua adição no software, evitando a quantificação de metanol por todo o processo de operação.

\subsection{Modelagem do Reator}

Uma lei cinética foi apresentada pelo autor em Esteban et al. (2015), onde foi utilizado o carbonato de potássio como catalisador, onde a reação tende naturalmente a atingir uma conversão de $100 \%$, tornando o equacionamento da equação reversa irrelevante.

A partir de tais resultados, foram escolhidos dois parâmetros com base no gráfico fornecidos na figura 1 , sendo eles: $1 \%$ em massa de catalisador, que forneceu bons resultados de velocidade de reação se comparado até mesmo com 1,25\% em massa, e a razão molar de 3 mols de DMC para 1 mol de glicerina, uma vez que essa quantidade aumenta a conversão do carbonato de glicerina.

Com esta cinética, o resultado obtido pelo software foi coerente com o apresentado na referência, como é possível observar na comparação da Figura 1.

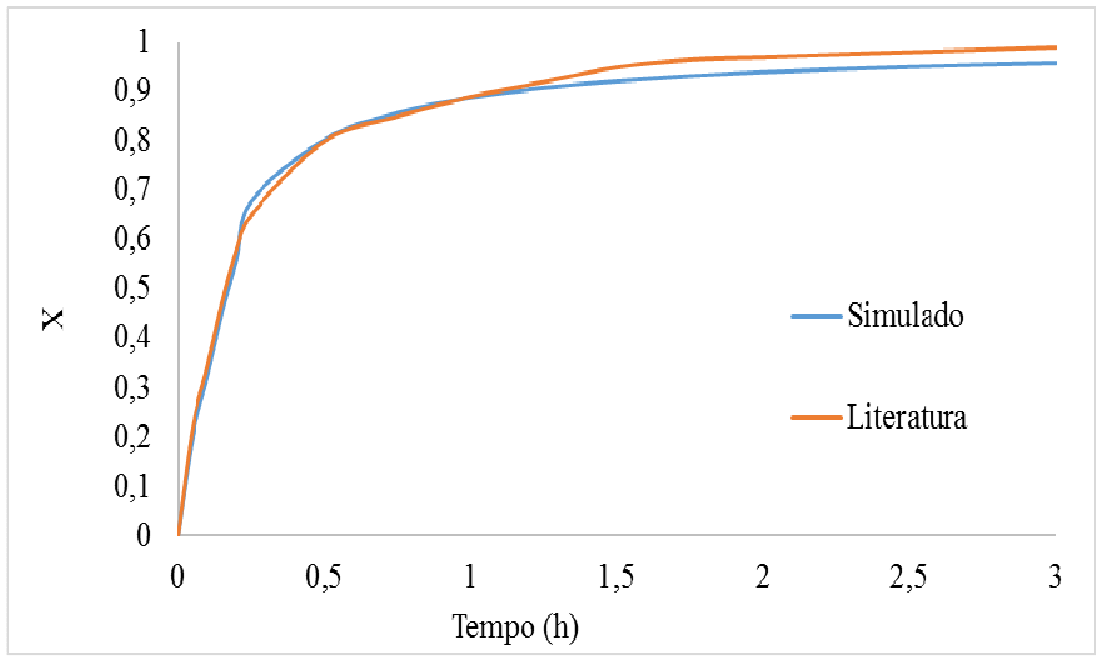

Figura 1 - Comparativo entre a curva adaptada da literatura e a simulada pelos autores $(\mathrm{X}$ representa a conversão)

\subsection{Construção do Sistema de Separação e Purificação}

Foram estudados três processos para a obtenção do GC, sendo o processo 1 sem reciclo, processo $2 \mathrm{com}$ reciclo e processo $3 \mathrm{com}$ reciclo e tanque flash. O conjunto total de equipamentos utilizados no projeto são um tanque de resina, três colunas de destilação e um tanque flash (Figura 2). O objetivo do tanque de resina é de retirar possíveis núcleofilos fortes em mistura, como o metóxido de sódio, que podem gerar a polimerização do produto dentro de um dos equipamentos em altas temperaturas. A primeira coluna de destilação teve como objetivo a separação entre a mistura total, metanol e parte do DMC que é carregado devido à interação química entre as substâncias. Na segunda coluna separou-se a mistura 
restante do excesso de substâncias classificadas como MONG e FAE que vêm da glicerina bruta alimentada ao processo. A última coluna retira a maior parte de DMC e deixa o GC à uma pureza maior que $90 \%$. Com a reciclagem do DMC para o ponto de mistura dos reagentes, é necessário o uso de um tanque flash para que o resto de DMC seja retirado do sistema até que sua concentração seja considerada baixa (da ordem de PPM).

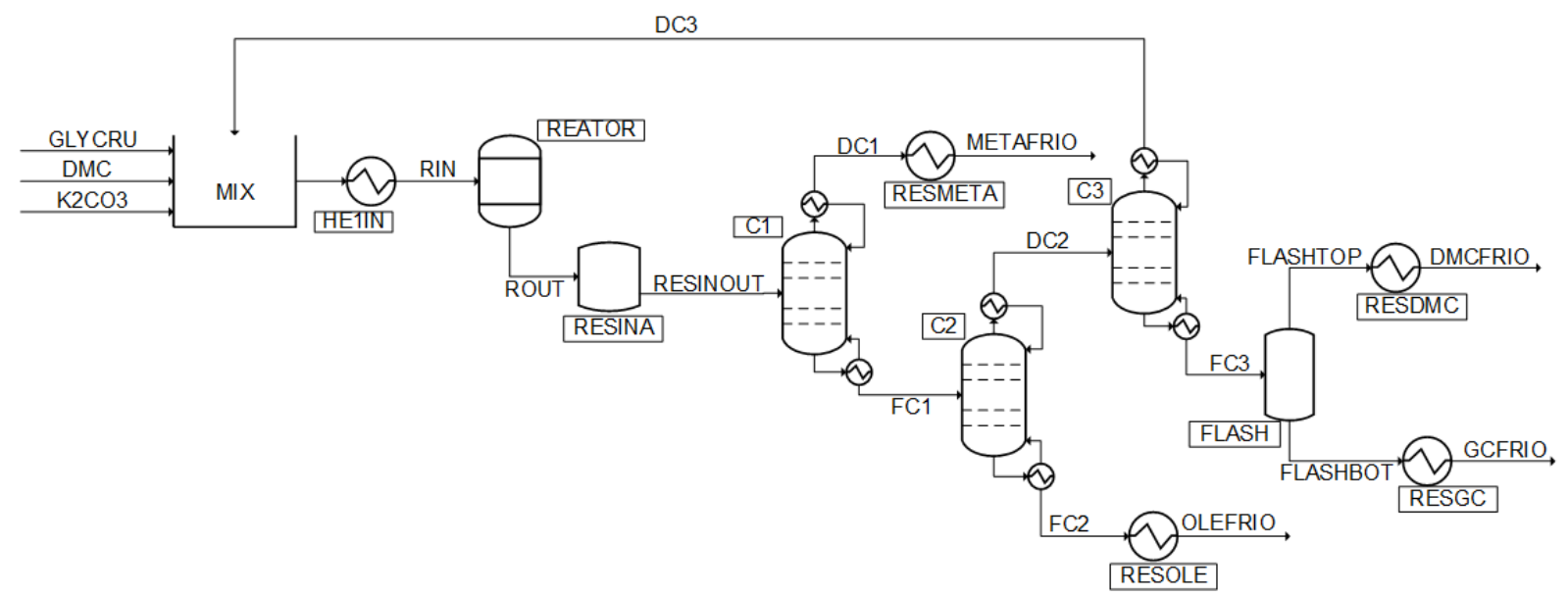

Figura 2 - Fluxograma do de blocos completo.

A pureza do produto final e a temperatura de fundo da terceira coluna de destilação são fortemente influenciadas pela conversão da glicerina, ocasionando a alta temperatura no reator. A partir de $72^{\circ} \mathrm{C}$ no reator forma-se vapores sendo necessária a pressurização de 2,5 bar para manter apenas uma fase líquida. Não foi de interesse o aumento do tempo de residência de 2 horas, pois a conversão aumentava insignificantemente em relação ao volume do reator, mas com tempos maiores necessitaria uma maior temperatura no reator

\subsection{Análise Econômica}

Foi realizada análise econômica para três tipos de processos distintos: sem reciclo (processo 1), com reciclo (processo 2) e com reciclo e flash (processo 3 ).

Foram calculados índices para auxiliar na comparação dos três processos, e estes são uma razão da massa de matéria prima, vapor e água de resfriamento pela massa de produto obtida no final do processo. Na tabela 1 são apresentados os índices calculados para os processos.

Tabela 1 - Índices obtidos a partir dos dados estimados para os processos.

\begin{tabular}{lrrr}
\hline Índices calculador & Processo [1] & Processo [2] & Processo [3] \\
\hline Matéria prima $\left(\mathrm{kg}_{\text {reagente }} \cdot \mathrm{kg}^{-1}\right.$ produto $)$ & 3,40 & 2,08 & 2,16 \\
Vapor $\left(\mathrm{kg}_{\text {vapor }} \cdot \mathrm{kg}^{-1}\right.$ produto $)$ & 4,20 & 4,45 & 4,72 \\
Água de resfriamento $\left(\mathrm{kg}_{\text {água }} \cdot \mathrm{kg}^{-1}{ }_{\text {produto }}\right)$ & 126,29 & 132,85 & 139,70 \\
Custo por massa de produto $\left(\mathrm{R} \$ \cdot \mathrm{kg}^{-1}\right)$ & 21,84 & 11,69 & 12,23 \\
\hline
\end{tabular}




\subsection{Proposta de Estratégia de Controle}

Após a realização da análise econômica dos três processos, considerou-se que o processo 1 não é viável por apresentar custos muito elevados, então optou-se em realizar a análise e construção de uma proposta de controle apenas para os processos 2 e 3, Figura 3.

Para o pré-aquecedor e resfriadores de fim de linha foram utilizados controladores de temperatura (TC) para manter a temperatura das linhas mudando a vazão de vapor e água de refrigeração. Os controladores de fluxo (FC) mantêm as correntes das linhas constantes controlando as válvulas. No misturador há um controlador de nível (LC) que mantém o nível do tanque por uma válvula.

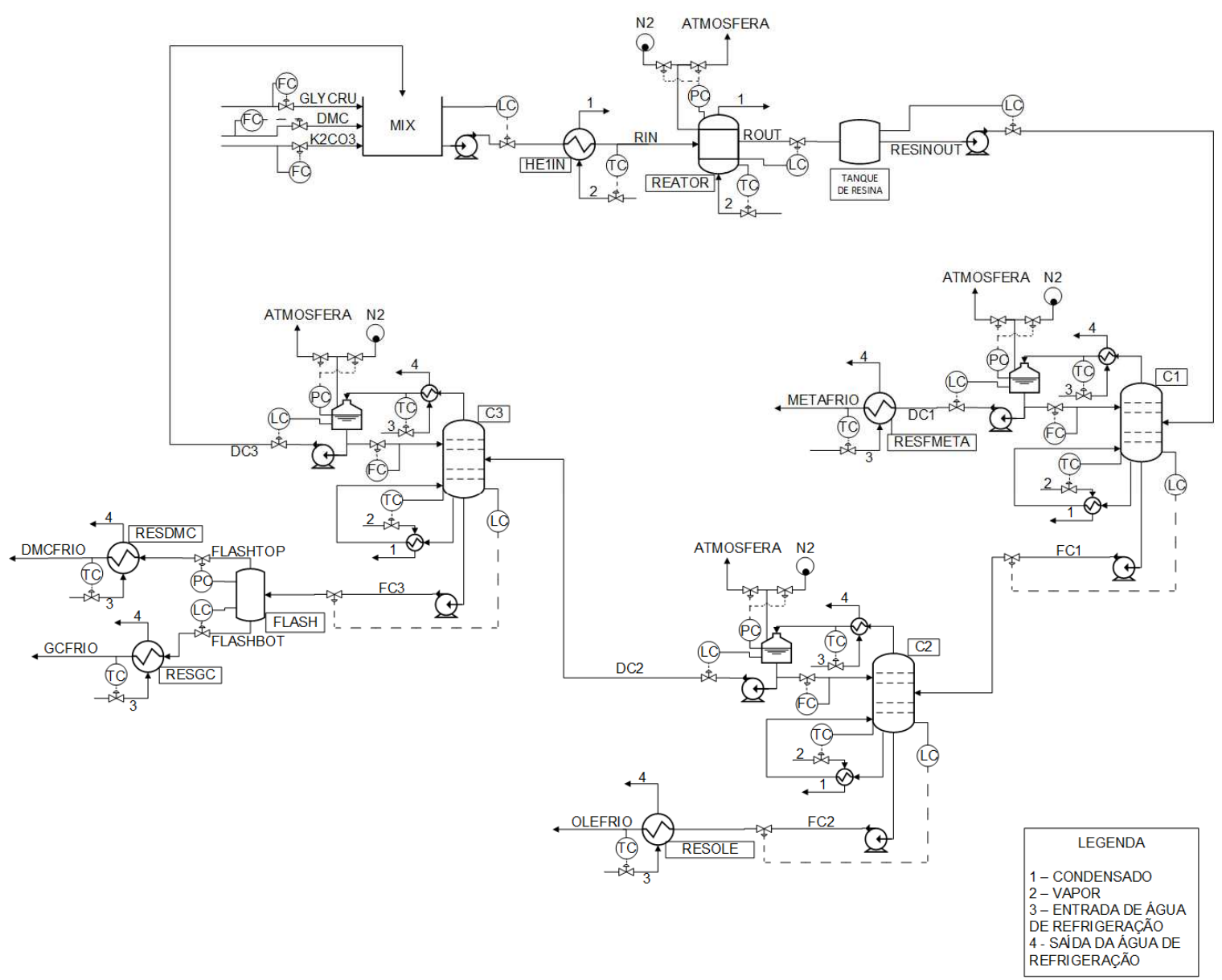

Figura 3 - Modelo de processo com propostas de controle para o processo com reciclo e com flash.

No reator só foi utilizado um TC com a finalidade de manter o sistema isotérmico e garantir a temperatura ideal para a reação. Com a formação de vapores devido a temperatura do reator, há a necessidade de um controlador PC, que regula a diminuição da pressão com um alívio, geralmente utilizando um flash, e um aumento de pressão com um inerte, geralmente $\mathrm{N}_{2}$, para manter uma única fase líquida no interior do reator e evitar um excesso de pressão. O LC tem a função de manter o nível do reator constante através de uma válvula na saída do reator. 
As colunas de destilação possuem a mesma proposta. No fundo das colunas possuem um LC em que regula a altura de líquido e manipula a válvula no fundo da coluna. No tanque de refluxo se apresenta o mesmo raciocínio para o seu LC, mas há um controle PC que regula a pressão da mesma maneira dita no reator. Para a vazão de refluxo utiliza-se um FC na linha.

Os trocadores de calor possuem um TC que ao medir a temperatura do final da troca de calor da linha, altera a quantidade de vapor ou água de refrigeração a ser alimentados. $\mathrm{O}$ reciclo utiliza um FC com o intuito de manter a vazão da linha constante, como representado na Figura 3.

\section{CONCLUSÃO}

Após a modelagem do reator pôde-se chegar a resultados satisfatórios, uma vez que a lei cinética utilizada no software Aspen Plus ${ }^{\circledR}$ ofereceu resultados muito próximos aos apresentados no gráfico de dados experimentais em Esteban et al. (2015), que está apresentado na figura 1. É importante citar que a temperatura do reator foi adotada constante $\left(100^{\circ} \mathrm{C}\right)$ para o controle do mesmo, considerando que para temperaturas maiores que $72^{\circ} \mathrm{C} \mathrm{o}$ metanol inicia sua vaporização, sendo necessária pressurização a 2,5 bar.

No processo 1 o GC é obtido ao final das etapas de purificação com uma fração mássica de $98 \%$, contudo, ao utilizar o reciclo (processo 2), esse valor diminui para 94,7\% depois de passar pelas mesmas etapas, sendo necessário mais uma operação unitária para aumentar a concentração de produto, o tanque flash (processo 3). Com a utilização do reciclo para diminuir o custo da matéria-prima houve um acréscimo no custo de operações, sendo necessária uma análise em relação ao custo do processo.

Pode-se dizer que os três processos apresentados representam de maneira consistente a produção de GC. O processo 1 tem como vantagens sua simplicidade estrutural e boa composição final do produto, com menos de $0,3 \%$ de DMC, substância que gera maior preocupação devido ao seu caráter tóxico, contudo, pelo uso excessivo de DMC e por possuir custo muito maior que os processos 2 e 3 (observado na tabela 1), esse processo pode ser desconsiderado.

O processo 2, com reciclo da matéria prima não reagida, apresenta boa reutilização das matérias primas, diminuindo a quantidade nova de reagentes necessários. A diminuição da quantidade de alimentação necessária de DMC segue os princípios da química verde, pois se trata de um reagente com caráter tóxico que não vai ser descartado e nem inserido no produto final. Mas apesar de reduzir o custo e reaproveitar matéria prima não utilizada, pode não ser uma opção viável devido à alta concentração de DMC no produto, um pouco maior do que $2,3 \%$.

Para solucionar este problema, foi proposto o processo 3, que possui reciclo aliado ao tanque flash, que alia a economia e reutilização de matéria prima garantida pelo reciclo e o tanque flash consegue purificar ainda mais o produto, deixando a composição final próxima do processo convencional apresentado primeiro. A principal desvantagem é a necessidade de uma operação unitária a mais, assim como maior gasto de utilidades, mas poderia ser uma alternativa viável. 


\title{
5 AGRADECIMENTOS
}

Ao Centro Universitário FEI pelo suporte para o desenvolvimento do trabalho.

\section{REFERÊNCIAS}

BIODIESELBR. Glicerina: Subproduto do biodiesel. Disponível em:

$<$ http://www.biodieselbr.com/biodiesel/glicerina/biodiesel-glicerina.htm>. Acesso em: 11 mar. 2016.

ESTEBAN, J. et. al. Phenomenological kinetic model of the synthesis of glycerol carbonate assisted by focused beam reflectance measurements. Chemical Engineering Journal, v. 260, p.434-443, 2015.

OCHOA-GÓMEZ.J.R.Et al. A Brief Review on Industrial Alternatives for the Manufacturing of Glycerol Carbonate, a Green Chemical. Organic Process Research \& Development 16(3) p.389-399.2012.

TENG, W. K.; NGOH, G. K.; YUSOFF, R.; AROUA, M. K.; Microwave-assisted transesterification of industrial grade crude glycerol for the production of glycerol carbonate. Chemical Engineering Journal, v.284, p.469-477, 2016.

\section{DESIGN OF NA INDUSTRIAL PROCESS FO GLYCERIN CARBONATE PRODUCION}

\author{
B. P. CELIBERTO ${ }^{1}$, C. C. FICO ${ }^{1}$, G. RUSSO ${ }^{1}$, L. L. NAME ${ }^{1}$, V. M. B. BARROS ${ }^{1}$, \\ N. L. FERREIRA ${ }^{1, *}$ \\ ${ }^{1}$ FEI University, Department of Chemical Engineering \\ *E-mail: nlibanio@fei.edu.br
}

\begin{abstract}
Biodiesel industries generates large amounts of glycerin what creates a demand for its suitable destination, with a possible transformation into a valuable product. One of these products is the glycerin carbonate. This substance has an increasing market as raw material for cosmetics, drugs, polymers and specilatiy solvents among others. This work shows an industrial process for the production of glycerin carbonate which achieves near complete conversion. The studies wre made with the use of the software Aspen Plus ${ }^{\circledR}$. All physical equilibrium properties were calculated by the NRTL method. A process flowsheet with basic control strategy is presented. It has been also made a preliminary economic analysis based on ultilities, raw materials and product costs
\end{abstract}

KEYWORDS: Glycerine; Transesterification; Catalysis; Glycerine carbonate. 